\title{
Electron Mobility in Nitride Materials
}

\author{
Clóves G. Rodrigues, ${ }^{1}$ Valder N. Freire, ${ }^{2}$, Áurea R. Vasconcellos, ${ }^{3}$ and Roberto Luzzi ${ }^{3}$ \\ ${ }^{1}$ Núcleo de Pesquisa em Física, Departamento de Física, Universidade Católica de Goiás, \\ Caixa Postal 86, 74605-010 Goiânia, Goiás, Brazil \\ 2 Departamento de Física, Universidade Federal do Ceará, Caixa Postal 6030, \\ Campus do Pici, 60455-760 Fortaleza, Ceará, Brazil \\ ${ }^{3}$ Instituto de Física Gleb Wataghin, Universidade Estadual de Campinas, \\ Caixa Postal 6165, 13083-970 Campinas, São Paulo, Brazil
}

Received on 23 April, 2001

\begin{abstract}
We contribute here a theoretical study of the electron mobility in $n$-doped GaN, InN, and AlN at moderate to high electric fields. We solve the set of coupled nonlinear integro-differential equations of evolution to obtain the steady-state values of the basic intensive nonequilibrium thermodynamic variables for the three materials. The regions with ohmic and non-ohmic behavior in the electron drift velocity dependence on the electric field strength are characterized in the three nitrides. The electron mobility is calculated, and it is shown that the larger corresponds to InN, and the smaller to $\mathrm{AlN}$.
\end{abstract}

The so-called nitride materials, like GaN, InN, and AlN, are presently object of intense research as a result of the large interest associated with application for blue/UV light emitting diodes and diode lasers (e. g. [1]). We contribute here a theoretical study consisting in an analysis of the electron mobility in $n$-doped samples of III-Nitrides - which are large direct-gap strongpolar semiconductors - at moderate to high electric fields. Basically we study transport phenomena which develop in the nonequilibrium thermodynamic state of the resulting "hot plasma" created by the presence of the electric field, mobile electrons moving in, and interacting with, the lattice and with impurities.

We study transport phenomena which develop in $n$-doped samples of $\mathrm{GaN}, \mathrm{InN}$, and $\mathrm{AlN}$, that is the change in the average energy of electrons and phonons, and the electronic current that ensues as a result of the presence of the electric field. This kind of studies are in general pursued with the help of Monte Carlo-like simulations; instead, we here resort to a powerful, concise, and soundly based kinetic theory for far-from equilibrium systems [2]. It is the one based on a nonequilibrium statistical ensemble formalism [3], the so-called MaxEnt-NESOM for short, which provides an elegant, practical, and physically clear picture for describing irreversible processes, as for example in far away from equilibrium semiconductors which is the case considered here. Moreover, we use the effective-mass approximation and therefore parabolic bands; this implies that in explicit applications it needs to be taken into account that there exists an upper limiting value for the electric field strength, corresponding to values such that intervalley scattering can be neglected. For numerical calculations we used the characteristic parameters shown in Table I.

Proceeding with the calculations we obtain the corresponding set of equations of evolution, namely

$$
\begin{gathered}
\frac{d}{d t} E_{e}(t)=-\frac{e F}{m_{e}^{*}} \cdot P_{e}(t)+J_{E}^{(2)}(t) \\
\frac{d}{d t} P_{e}(t)=-n V e F+J_{P_{e}}^{(2)}(t)+J_{P_{e, i m p}}^{(2)}(t) \\
\frac{d}{d t} E_{L O}(t)=-J_{E_{L O}}^{(2)}(t)-J_{L O, A N}^{(2)}(t) \\
\frac{d}{d t} E_{A C}(t)=-J_{E_{A C}}^{(2)}(t)+J_{L O, A N}^{(2)}(t)-J_{A C, d i f}^{(2)}(t) .
\end{gathered}
$$

(all $J^{(2)}$ being positive or null only when equilibrium is attained). $E_{e}$ is the electrons' energy and $P_{e}$ their linear momentum; $E_{L O}$ the energy of the LO phonons, which strongly interact with the carriers via Fröhlich potential in these strong-polar semiconductors; $E_{A C}$ is the energy of the acoustic phonons, which play a role of a thermal bath; and $F$ stands for the constant electric field in the $x$-direction. 
Table I. Parameters of $n$-doped wurtzite $\mathrm{AlN}$, InN, and GaN used in the numerical solution of the transport equations.

Parameter
Electron effective mass $\left(m_{0}\right)$
Lattice Constant a $(\AA)$
Lattice Constant c $(\AA)$
LO-Phonon Energy $\hbar \omega_{L O}(\mathrm{meV})$
Optical Dielectric Constant $\epsilon_{\infty}$
Static Dielectric Constant $\epsilon_{0}$
Mass Density $\left(\mathrm{g} / \mathrm{cm}^{3}\right)$
Sound Velocity $\left(\times 10^{5} \mathrm{~cm} / \mathrm{s}\right)$
Acoustic Def. Potential $\mathrm{E}_{1 e}(\mathrm{eV})$
Piezoelectric Constant $\mathrm{h}_{P Z}\left(\mathrm{C} / \mathrm{m}^{2}\right)$

$\begin{array}{ccc}\text { AlN } & \text { InN } & \text { GaN } \\ 0.35^{(a)} & 0.11^{(d)} & 0.22^{(a)} \\ 3.11^{(b)}(b) & 3.54^{(e)} & 3.189^{(f)} \\ 4.98^{(b)} & 5.7^{(e)} & 5.19^{(f)} \\ 99.2^{(b)} & 89^{(e)} & 92^{(g)} \\ 4.77^{(b)} & 8.4^{(e)} & 5.35^{(f)} \\ 8.5^{(b)} & 15.5^{(e)} & 9.5^{(f)} \\ 3.23^{(b)} & 6.81^{(e)} & 6.09^{(h)} \\ 6.04^{(b)} & 4.16^{(b)} & 4.40^{(b)} \\ 9.5^{(b)} & 7.1^{(e)} & 8.3^{(i)} \\ 0.92^{(c)} & 0.375^{(e)} & 0.375^{(i)}\end{array}$

(a) K. Kim et al., Phys. Rev. B 56, 7363 (1997); (b) V. W. L. Chin et al., J. Appl. Phys. 75, 7365 (1994); (c) S. K. O'Leary et al., Solid State Communic. 105, 621 (1998); (d) Y. C. Yeio et al., J. Appl. Phys. 75, 1429 (1998); (e) W. M. Yim et al., J. Appl. Phys. 44, 292 (1973); (f) S. Strite and H. Morkoç, J. Vac. Sci. Technol. B 10, 1237 (1992); $(g)$ A. S. baker and M. Ilegems, Phys. Rev. B 7, 743 (1973); ${ }^{(h)}$ H. Hahn and R. Juza, Z. Allg. Anorg. Chem. 244, 111 (1940); ${ }^{(i)}$ M. Shur et al., J. Elec. Mat. 25, 777 (1996).

Let us analyze these equations term by term. In Eq. (1) the first term on the right accounts for the rate of energy transferred from the electric field to the carriers, and the second term accounts for the transfer of the excess energy of the carriers - received in the first term - to the phonons. In Eq. (2) the first term on the right is the driving force generated by the presence of the electric field. The second term is the rate of momentum transfer due to interaction with the phonons, and the last one is a result of scattering by impurities. In Eq. (3) and Eq. (4) the first term on the right describes the rate of change of the energy of the phonons due to interaction with the electrons. More precisely they account for the gain of the energy transferred to then from the hot carriers and then the sum of contributions $J_{E_{L O}}^{(2)}$ and $J_{E_{A C}}^{(2)}$ is equal to the last in Eq. (1), with change of sign. The second term in Eq. (3) accounts for the rate of transfer of energy from the optical phonons to the acoustic ones via anharmonic interaction. The contribution $J_{L O, A N}^{(2)}$ is the same but with different sign in Eq. (3) and (4). Finally, the diffusion of heat from the $\mathrm{AC}$ phonons to the reservoir is account for in the last term in Eq. (4). The detailed expressions for the collision operators are given in Ref. [4].

Proceeding to the solution of the coupled set of nonlinear integro-differential equations, Eqs. (1) to (4), using the parameters of Table I, a concentration of carriers $n=10^{17} \mathrm{~cm}^{-3}$ and a reservoir temperature of 300 $\mathrm{K}$, we can derive the mobility, $\mathcal{M}$ of the carriers, as given by

$$
\mathcal{M}=\frac{\left|\mathbf{v}_{e}\right|}{|\mathbf{F}|}
$$

with the drift velocity $\mathbf{v}_{e}$ related to linear momentum per electron by

$$
\mathbf{P}_{e}=m_{e}^{*} \mathbf{v}_{e} .
$$

In Fig. 1 the mobility in terms of the electric field strength is shown. It can be noticed that the larger mobility corresponds to InN, which can be ascribed to the electrons having a smaller effective mass in $\mathrm{InN}$ than in GaN and AlN.

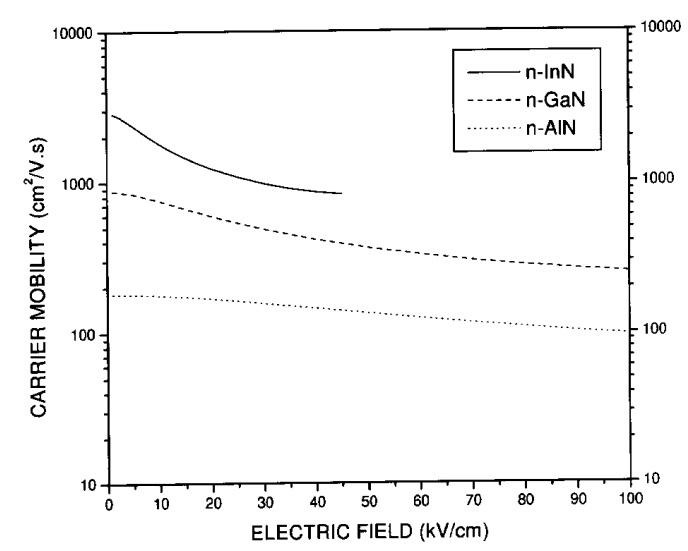

Figure 1. Dependence of the carrier mobility on the electric field.

In Figs. 2a, b and c can be seen the dependence of the drift velocity for the three materials with the electric field strength. As already noticed in the case of the mobility, the smaller the carrier effective mass the larger is the drift velocity (the current). At low fields, an Ohmic region is present, however, limited to fields of, roughly, $20 \mathrm{kV} / \mathrm{cm}, 7 \mathrm{kV} / \mathrm{cm}$, and $3 \mathrm{kV} / \mathrm{cm}$ for $\mathrm{AlN}$, $\mathrm{GaN}$, and InN, respectively. Beyond this value there 
follows a departure from the Ohmic behavior (notice the break in scale in the horizontal axis).
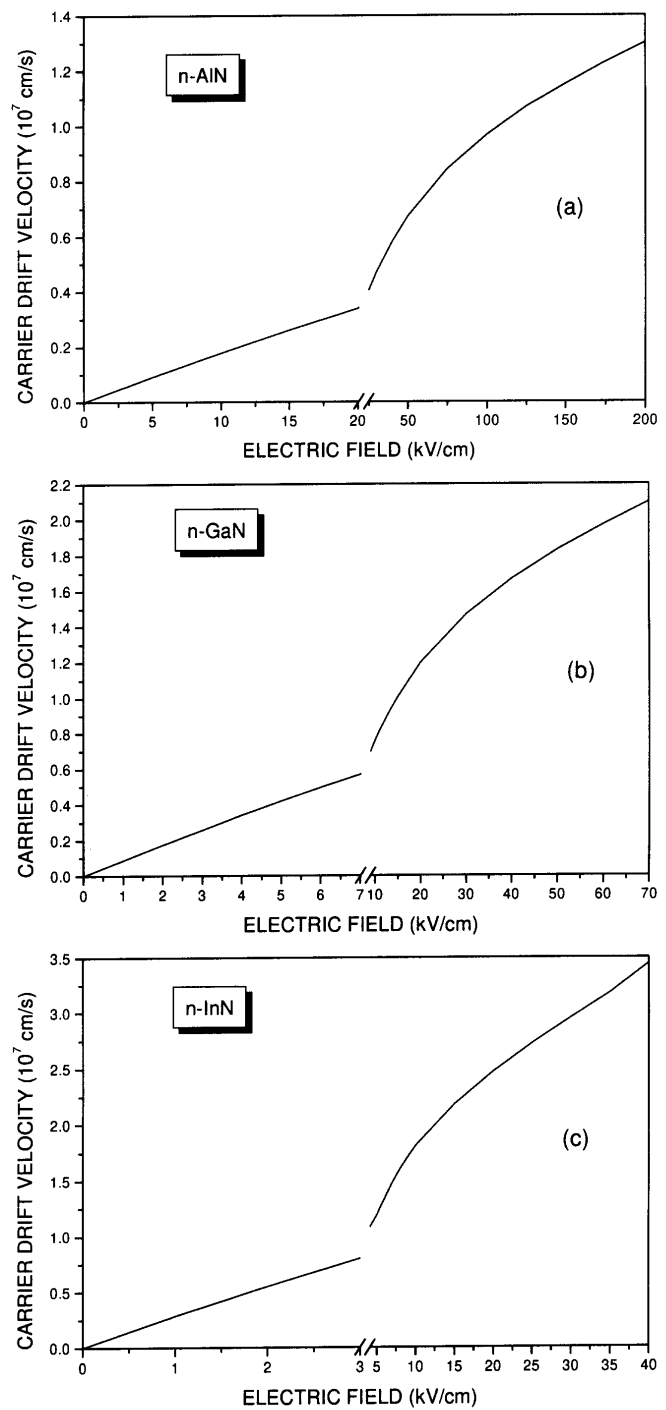

Figure 2. Steady-state drift velocity of the carriers as a function of the electric field.

In conclusion, we have presented a study on the transport characteristics (focusing on the electron mobility and ohmic/non-ohmic behavior of the electron drift velocity) of wurtzite GaN, AlN, and InN using quantum transport equations derived from the nonequilibrium statistical ensemble formalism. The regions with ohmic and non-ohmic behavior in the electron drift velocity dependence on the electric field strength were characterized in the three nitrides. The electron mobility was calculated, and it was shown that the larger corresponds to InN, and the smaller to AlN.

Acknowledgement - The authors acknowledge partial financial support received from the São Paulo State Research Agency (FAPESP), the Brazilian National Research Council (CNPq), the Funding Agency of the Ceará State (FUNCAP), and the Brazilian Ministry of Planning through FINEP; the authors ARV, VNF, RL are CNPq Research Fellows.

\section{References}

[1] S.J. Pearson, ed. articles in GaN and Related Materials, in the series Optoelectronic Properties of Semiconductors and Superlattices (Gordon and Breach, New York, 1997).

[2] L. Lauck, A.R. Vasconcellos, and R. Luzzi, Physica A 168 , 789 (1990); C.G. Rodrigues, A.R. Vasconcellos, and R. Luzzi, Transp. Theor. Stat. Phys. 29, 733 (2000).

[3] R. Luzzi and A.R. Vasconcellos, Fortsch. Phys./Prog. Phys. 38, 887 (1990); R. Luzzi, A.R. Vasconcellos, and J.G. Ramos, Predictice Statistical Mechanics: A Nonequilibrium Statistical Ensemble Formalism, (Kluwer Academic, Dordrecht, in press); also J. Mod. Phys. B 14, 3189 (2000), and e-Print http://xxx.lanl.gov/cond-mat/9909160.

[4] C.G. Rodrigues, Ph.D. Thesis (Unicamp, Campinas, SP, Brasil, 2001), and forthcoming papers; also C.G. Rodrigues, A. R. Vasconcellos, and R. Luzzi, J. Transp. Theor. Stat. Phys. 29, 733 (2000). 